\title{
Projected Changes in the Seasonal Cycle of Surface Temperature
}

\author{
JOHN G. DWYER \\ Department of Applied Physics and Applied Mathematics, Columbia University, New York, New York \\ Michela BiasutTi \\ Lamont-Doherty Earth Observatory, Columbia University, Palisades, New York
}

ADAM H. SOBEL

Department of Applied Physics and Applied Mathematics, Department of Earth and Environmental Sciences, and Lamont-Doherty Earth Observatory, Columbia University, New York, New York

(Manuscript received 21 December 2011, in final form 2 April 2012)

\begin{abstract}
When forced with increasing greenhouse gases, global climate models project a delay in the phase and a reduction in the amplitude of the seasonal cycle of surface temperature, expressed as later minimum and maximum annual temperatures and greater warming in winter than in summer. Most of the global mean changes come from the high latitudes, especially over the ocean. All 24 Coupled Model Intercomparison Project phase 3 models agree on these changes and, over the twenty-first century, average a phase delay of 5 days and an amplitude decrease of $5 \%$ for the global mean ocean surface temperature. Evidence is provided that the changes are mainly driven by sea ice loss: as sea ice melts during the twenty-first century, the previously unexposed open ocean increases the effective heat capacity of the surface layer, slowing and damping the temperature response. From the tropics to the midlatitudes, changes in phase and amplitude are smaller and less spatially uniform than near the poles but are still prevalent in the models. These regions experience a small phase delay but an amplitude increase of the surface temperature cycle, a combination that is inconsistent with changes to the effective heat capacity of the system. The authors propose that changes in this region are controlled by changes in surface heat fluxes.
\end{abstract}

\section{Introduction}

On annual and longer time scales the seasonal cycle is responsible for around $90 \%$ of the total surface temperature variance. In this study we focus on potential changes in the seasonality of the surface temperature due to expected increases in greenhouse gases. These are distinct from changes due to the mean temperature increase, even though the latter can also affect the seasonality of phenomena linked to specific climate thresholds, such as streamflow timing due to melting snow (Stewart et al. 2005) and plant flowering (Fitter and Fitter 2002). Here we concentrate on changes to the phase and amplitude of the annual cycle in surface temperature

Corresponding author address: John G. Dwyer, Department of Applied Physics and Applied Mathematics, Columbia University, 500 W. 120th St., New York, NY 10027.

E-mail: jgd2102@columbia.edu (and to a lesser extent, temperature in the upper atmosphere), independent of the annual mean warming. Specifically, we are interested in the geographic pattern of the response in phase and amplitude to greenhouse gases and the mechanisms responsible for these changes.

In the models of the World Climate Research Programme (WCRP) Coupled Model Intercomparison Project phase 3 (CMIP3) multimodel dataset (Meehl et al. 2007), the main changes in seasonality of surface temperature are a robust delay in phase and a robust decrease in amplitude, where we take "robust" to mean that the changes occur in all or nearly all of the models. This means that the models predict peak temperatures to occur later in the year and the difference between annual maximum and minimum temperatures to shrink. We illustrate these effects in Fig. 1 by plotting the hemispheric, multimodel-mean 2-m surface air temperature over the ocean for the last two decades of the twentieth and twenty-first centuries with the annual mean removed. By 

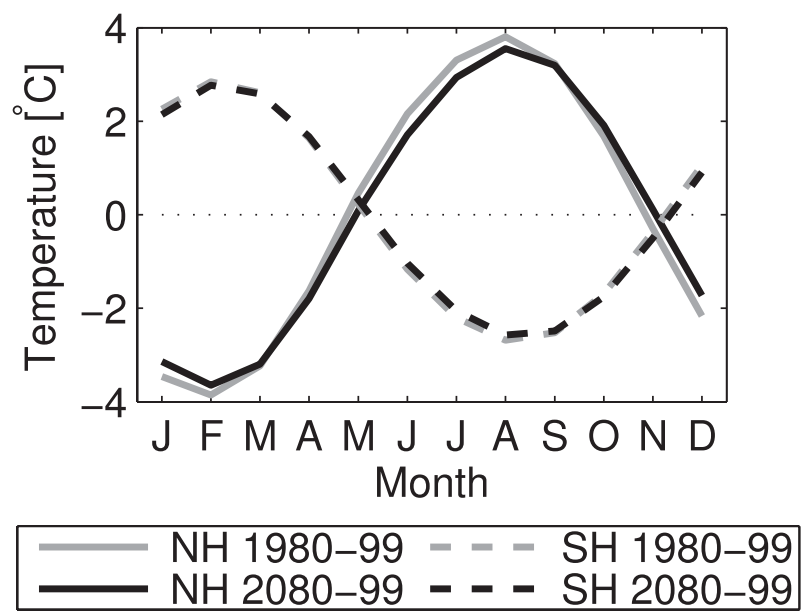

FIG. 1. Hemispherically averaged, multimodel mean monthly surface air temperature anomaly $\left({ }^{\circ} \mathrm{C}\right)$ over ocean for the last two decades of the twentieth (gray) and twenty-first (black) centuries. Both Northern (solid) and Southern (dashed) Hemispheres have a phase delay and amplitude decrease.

fitting the anomalies to sinusoids we can quantify the changes compared to the late twentieth century: the temperature cycle in the late twenty-first century has a phase delay of 6 days in the Northern Hemisphere and 3 days in the Southern Hemisphere and an amplitude decrease of $6 \%$ in the $\mathrm{NH}$ and $3 \%$ in the $\mathrm{SH}$.

As we will show in section 4 , the dominant component of the global mean response is a strong phase delay and amplitude reduction over the high-latitude ocean. Manabe and Stouffer (1980), Manabe et al. (1992), and Mann and Park (1996) noticed this high-latitude signal in earlier generations of climate models and proposed that it was a consequence of an increase in effective heat capacity due to sea ice loss. Sufficiently thick sea ice insulates the atmosphere from the ocean and curtails heat storage in the climate system. As the ice thins and melts, the insulation weakens and disappears and the effective heat capacity of the surface increases. Due to this additional thermal inertia, the temperature responds more slowly and with a smaller amplitude than it would were the ice present.

We build on the earlier modeling studies by demonstrating the seasonality changes in the most recent generation of climate models, investigating the spatial patterns of seasonality changes, and providing evidence that sea ice is driving the high-latitude seasonality changes in the models. To verify this mechanism, we interpret the CMIP3 results in the context of a simple energy balance model for surface temperature. Using this and other tools we show that the high-latitude phase delay and amplitude reduction are consistent with an increased effective heat capacity and inconsistent with other potential mechanisms including changes in the seasonality of surface heat fluxes or heat transport. Furthermore, we link the effective heat capacity changes to sea ice loss quantitatively.

Previous observational studies in the $\mathrm{NH}$ midlatitudes have found a phase advance driven by changes over land and an amplitude reduction during the second half of the twentieth century (Thomson 1995; Mann and Park 1996; Stine et al. 2009) and have questioned the ability of the CMIP3 models to reproduce the observed phase and amplitude variations. More recent work by the same authors suggest that the small seasonality changes over land might be due to natural variability in atmospheric circulation (Stine and Huybers 2012), in which case we would not expect the multimodel mean to match such changes. Over the same period, Stine and Huybers found a nonstatistically significant phase delay and an amplitude reduction in the NH midlatitudinal oceans, and recent studies of surface temperature over the Arctic Ocean also found evidence of a phase delay and amplitude decrease due to strong late fall and early winter warming during the last 20-30 years (Serreze et al. 2009; Screen and Simmonds 2010). The correspondence between Arctic sea ice loss over the last few decades (Stroeve et al. 2007) and local changes in seasonality suggests that a key mechanism for the simulated late twenty-first-century seasonality changes is also present in nature.

In the tropics and subtropics there is a smaller, yet still robust, change in the temperature seasonality, different in nature from the high-latitude signal. There the CMIP3 models project a small phase delay and an amplitude increase, the latter being opposite in sign to the high-latitude amplitude response. Because the phase and amplitude changes are of the same sign, these lowlatitude changes cannot be primarily driven by a change in effective heat capacity, as will be shown below. Instead, some other mechanism must be the primary cause. We provide evidence that changes in the seasonality of surface flux are linked to the low-latitude temperature phase delay and amplitude increase. The source of the low-latitude changes in fluxes is not clear, but it might be wind speed changes, which Sobel and Camargo (2011) argued were responsible for the amplitude increase. The seasonality changes in temperature, though small, could be related to seasonality changes in the onset and demise of the monsoons (Biasutti and Sobel 2009; Seth et al. 2011), especially given the sensitivity of the ITCZ to the tropical SST distribution (e.g., Chiang et al. 2002).

The rest of the paper is laid out as follows. In the next section we give background information on the data that we analyze from CMIP3 and a reanalysis dataset and 
explain the methods used to calculate the phase and amplitude of the annual cycle. In section 3 we describe the climatological structure of the annual cycle at the surface and aloft, as represented by both the CMIP3 multimodel mean and the reanalysis, and demonstrate agreement between the two, as both capture the slow, weak surface temperature response to insolation over the ocean and the fast, strong response over land. Moreover, both datasets show that over sea ice, the temperature response is more landlike than oceanlike. In section 4 we detail the changes to the annual cycle at the surface and aloft as projected by the models and discuss the differences at high and low latitudes. In section 5 we look at both of these regions individually and demonstrate that the changes in sea ice account for much of the high-latitude temperature cycle change, while changes in the seasonality of surface flux explain the seasonal temperature changes. Finally, we summarize our findings in section 6 .

\section{Data and methods}

Throughout this study we use the CMIP3 twentiethcentury historical simulations [Twentieth-Century Climate in Coupled Model (20C3M)] and twenty-first-century A1B scenario simulations, where atmospheric $\mathrm{CO}_{2}$ reaches 700 ppm by 2100 (Meehl et al. 2007). Monthly temperature data is sufficient to characterize the phase and amplitude of the annual cycle. We use only one realization of each model. All 24 models store temperature data at all levels, but only 20 models store sea ice data and 18 store total surface flux data. When data is missing, we take the multimodel mean to be the subset of models with available data. Surface temperature is defined as the 2-m air temperature, which is tightly constrained by surface fluxes to be close to SST over open ocean, though not over sea ice (since SST is constrained to the freezing point of seawater). We compare the model results with the 40-yr European Centre for Medium-Range Weather Forecasts Re-Analysis (ERA40) dataset (Uppala et al. 2005), which covers 1958 2001. The reanalysis assimilates satellite and terrestrial observations using a climate model. Where observations are relatively sparse, like the Arctic Ocean, comparison between the CMIP3 models and the reanalysis are not as informative as in other regions.

We calculate the phase of the seasonal cycle using two different techniques. The first uses empirical orthogonal functions (EOFs). In this approach we decompose the climatological mean, monthly data into spatial eigenfunctions of the covariance matrix and associated principal component time series (PCs) (Kutzbach 1967). We obtain amplitude and phase information by fitting a sinusoid to the PC representing the annual cycle, which is always associated with the EOF capturing the highest fraction of the total variance, except within about $5^{\circ}$ of the equator. The other method is Fourier transformation of the data to obtain the annual harmonic of each field of interest. Both methods are able to resolve phase and amplitude precisely from monthly data. Fourier transforms can be calculated pointwise, but they cannot obtain reliable phase information in the tropics because of the relatively small amplitude of the annual cycle there. EOFs are defined for the entirety of the domain of interest but are dominated by regions of large annual variance. After spatially averaging area-weighted phases and amplitudes calculated with a Fourier transform, the results are nearly identical to those calculated using EOFs over the same domain.

Since our analysis is predicated on temperature cycles being accurately described by a sinusoid with a period of 1 year, we will only use locations for which its annual component explains at least $80 \%$ of the total variance (we loosen this restriction to $70 \%$ when we plot the annual cycle of surface flux so that the two can be compared in the same regions). These are roughly the same regions for which insolation is dominated by the annual harmonic (Trenberth 1983). Surface temperature and insolation each have over $95 \%$ of their total variance described by the annual cycle between $20^{\circ}$ and $70^{\circ}$ latitude. At higher latitudes only around $85 \%$ of the insolation is due to the annual cycle owing to the sunless winters and nightless summers. Over Antarctica, the temperature cycle has a large semiannual component due to the "coreless winters" of relatively constant cold temperatures owing to the large landmass being in longwave radiative balance as well as to dynamical effects (Loon 1967). In the Arctic, the temperature cycle is surprisingly annual with over $95 \%$ of the total temperature variance described by the first harmonic. The strength of the annual harmonic of temperature in the Arctic can be partly attributed to the seasonal sea ice cycle, which is not discrete but instead smoothly varies throughout the year with advancing and retreating ice margins, thickening and thinning sheet ice, melt pond formation, and other effects (Eicken 2003). In the tropics, the sun passes overhead 2 times per year and the second harmonic becomes prominent for both insolation and temperature: the variance explained by the annual cycle drops below $50 \%$ for the insolation and below $70 \%$ for temperature near the equator.

The earth's axial and apsidal precession also changes the phase of the temperature cycle toward earlier seasons in the $\mathrm{NH}$ and later seasons in the $\mathrm{SH}$ (Stine and Huybers 2012). Only four of the CMIP3 models have a different phase of insolation between the twentieth and twenty-first centuries. We account for any such 
(a) ERA-40 Phase

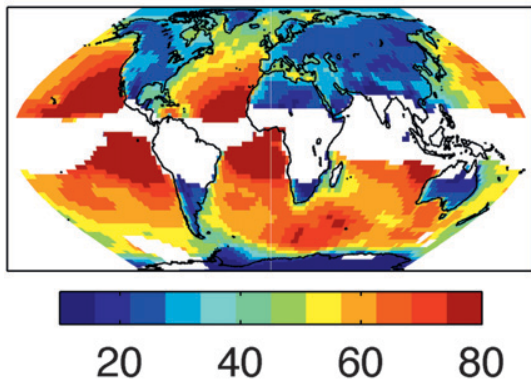

(d) ERA-40 Amplitude

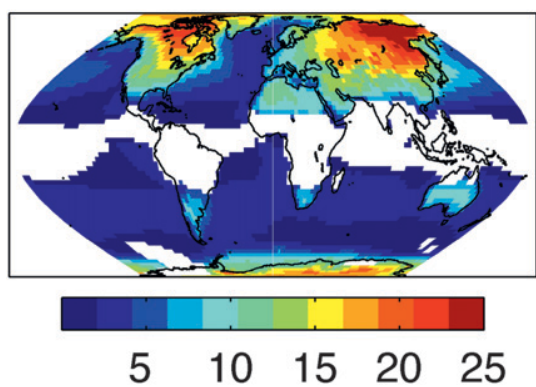

(b) CMIP3 Phase

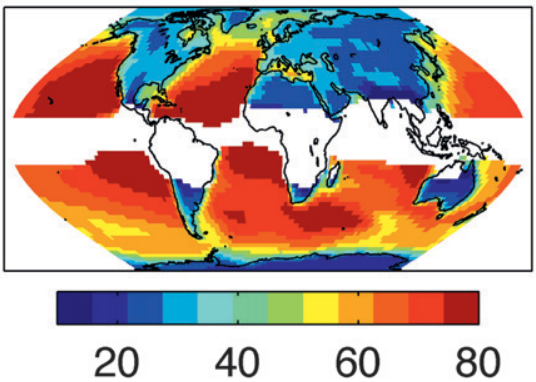

(e) CMIP3 Amplitude

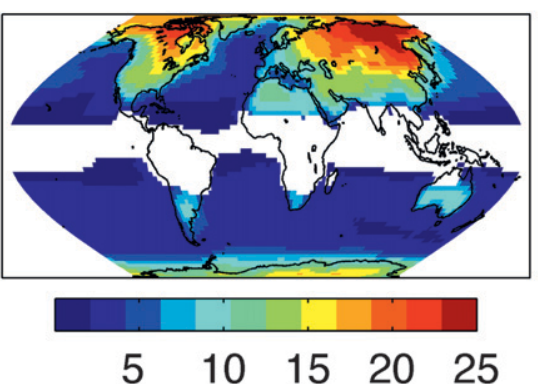

(c) CMIP3 - ERA-40 Phase

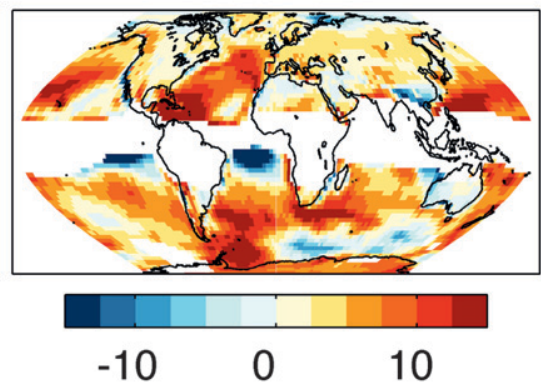

(f) CMIP3 - ERA-40 Amp.

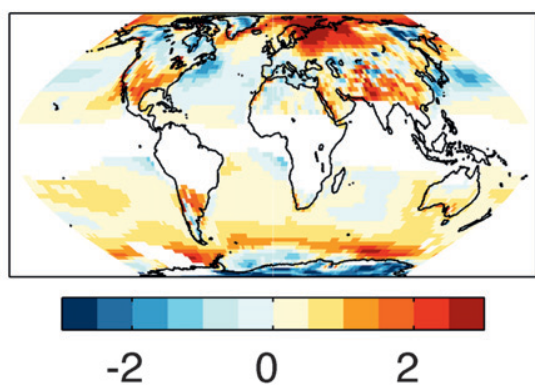

FIG. 2. The 1958-2001 mean temperature phase (days) from insolation for (a) the ERA-40, (b) the CMIP3 multimodel mean, and (c) their difference. The mean amplitude $\left({ }^{\circ} \mathrm{C}\right.$ ) over the same period for (d) ERA-40, (e) CMIP3, and (f) their difference. Places where the annual cycle does not represent at least $80 \%$ of the total variance are not plotted.

changes by measuring the temperature phase relative to the local insolation phase so that any phase changes in the models are not due to celestial mechanisms.

\section{Climatological structure}

Before analyzing the changes to the annual cycle, we look at the long-term mean of the phase and amplitude at the surface and aloft for both the multimodel mean and the reanalysis.

\section{a. Surface}

The seasonality of incoming diurnal mean solar radiation depends only on latitude. The phase of annual insolation is a weak function of latitude, varying by only a few days between the tropics and poles, but the amplitude of annual insolation increases markedly with latitude from about $50 \mathrm{~W} \mathrm{~m}^{-2}$ at $10^{\circ}$ to around $275 \mathrm{~W} \mathrm{~m}^{-2}$ at $90^{\circ}$ (Trenberth 1983). Since the temperature cycle is primarily governed by the solar cycle, the seasonality of temperature has a pattern that is qualitatively similar to that of insolation but with substantial departures due to the local effective heat capacity of the surface layer.

Effective heat capacity of the surface is a function of both the material properties and dynamical behavior of the layer adjacent to the atmosphere. We refer to it as effective since it is neither the intensive heat capacity (per unit mass) of some material substance nor the extensive heat capacity of a fixed mass of that substance. Rather, it is the heat capacity of the layer of material through which heat is transported sufficiently rapidly that it is influenced by the atmosphere on time scales of interest. The ocean mixed layer has a relatively large heat capacity because turbulent mixing transports heat downward so that a thick layer of water is rapidly influenced by surface fluxes. This causes the ocean surface temperature to respond sluggishly and with small amplitude to heat fluxes at the ocean surface. Temperature has a much faster and stronger response to insolation over land than over ocean because only a very thin layer of the land responds on annual time scales since the primary soil heat transfer process is diffusion with a small diffusivity. The effective heat capacity of land depends to some extent on the type of soil and the moisture content, but a typical estimate would be roughly equivalent to a 2-m oceanic mixed layer depth (Carson and Moses 1963), though this does not account for the presence of rivers and lakes. For comparison, the heat capacity of an atmospheric air column is roughly equivalent to that of $4 \mathrm{~m}$ of ocean.

We plot the ERA-40 and CMIP3 multimodel-mean surface temperature phase lag from insolation averaged over 1958-2001 in Figs. 2a and 2b. The models show 
good fidelity to the reanalysis in their geographic structure. Phase delays are smaller over the continents, as temperature over land responds more quickly than over ocean, and this effect is propagated downwind [the temperature phase in the $\mathrm{NH}$ midlatitudes can be well described by the westward distance from the coast (Stine et al. 2009)]. The largest differences between models and the reanalysis are mainly over the midlatitude oceans where the models have a larger phase lag than those of ERA-40 (Fig. 2c) for reasons unknown.

In regions of sea ice (e.g., the high-latitude Arctic and Southern Ocean), the phase lag has a response in between those of land and ocean. Around the maximal winter extent ice margins, the temperature responds slowly, as over the ocean, while closer to the poles the temperature response is more akin to that over land for both the reanalysis and models. Since the observational record is limited in the high-latitude oceans, the reanalysis should not be viewed as a strong constraint on the CMIP3 results there. This pattern is consistent with the insulating effect of sea ice becoming stronger in regions of more extensive and thicker ice coverage, and being responsible for the rapid polar temperature response due to a reduced effective heat capacity.

A similar pattern holds for the amplitude. Figures $2 d$ and 2e show the temperature amplitude from the ERA40 and the CMIP3 multimodel mean. Both show that most of the surface has a relatively weak seasonal cycle with an amplitude under $5^{\circ} \mathrm{C}$. The cycle is much stronger over land and sea ice. The difference between models and the ERA-40 is plotted in Fig. 2f. Differences are mostly small, though the multimodel mean has a larger amplitude in most places.

We provide more evidence that effective heat capacity sets the climatological surface temperature phase and amplitude and that the ice-covered ocean has a similar heat capacity to that of land in Fig. 3. In both Figs. 3a and $3 \mathrm{~b}$, we plot the percentages of land and sea ice that comprise each zonal band as a function of latitude. In Fig. 3a we plot the zonal mean temperature phase, while in Fig. $3 b$ we plot zonal mean temperature amplitude divided by insolation amplitude. The phase is strongly anticorrelated with the fraction of land and sea ice $(r=-0.85)$, while the amplitude is strongly correlated $(r=0.83)$, as we expect from the different effective heat capacities of ocean and land or sea ice. If sea ice is not included, correlations of the land fraction drop to $r=-0.64$ with phase but hold steady at $r=0.85$ with amplitude, suggesting that icecovered ocean has a landlike effective heat capacity.

\section{b. Aloft}

The zonal mean temperature phase aloft as a function of latitude and pressure is plotted in Figs. $4 \mathrm{a}$ and $4 \mathrm{~b}$ for
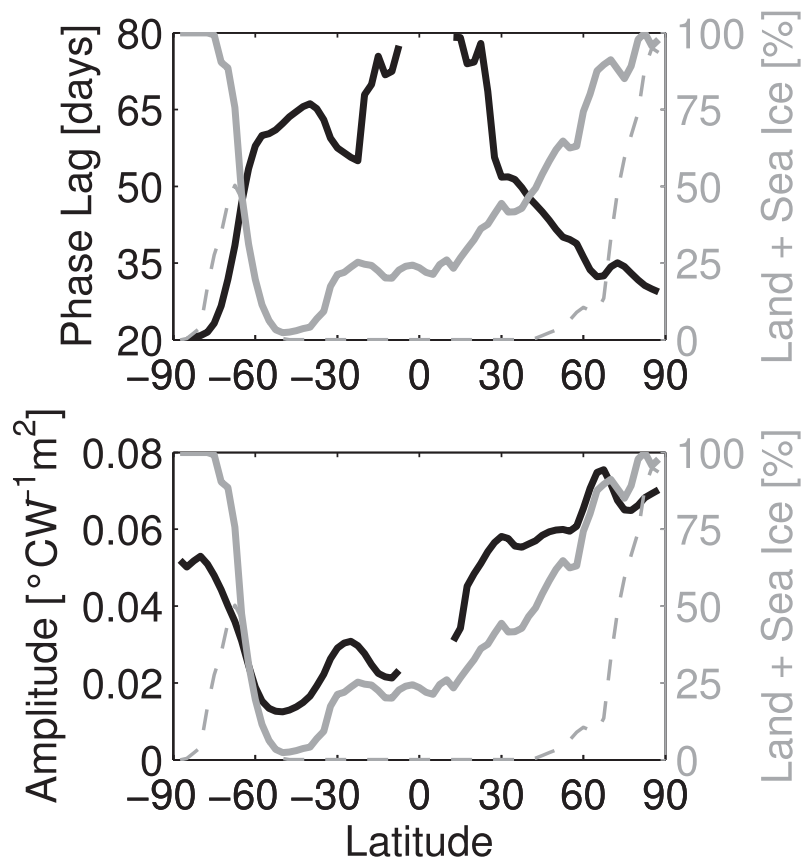

FIG. 3. (top) Zonal mean surface temperature phase lag from insolation and (bottom) amplitude divided by insolation amplitude are in black. In both panels the percentage of each latitude band made up of land or sea ice (thick, solid) and sea ice alone (thin, dashed) are in gray. The data are for the CMIP3 multimodel mean from 1900 to 1960 but are representative of observations as well. Phase and amplitude both correlate strongly with the amount of land and sea ice ( $r=-0.85$ and $r=0.83$, respectively).

the ERA-40 and the CMIP3 multimodel mean. While the two exhibit some differences, they have similar overall structures. For much of the troposphere the phase lag stays roughly constant with height above the boundary layer, presumably reflecting vertical mixing from the surface. Figure $4 \mathrm{c}$ shows the difference in phase lag between models and the reanalysis. Most locations differ by less than 5 days.

Figures $4 \mathrm{~d}$ and $4 \mathrm{e}$ show the corresponding plots for the amplitude. Both the reanalysis and models have a very different amplitude structure between the $\mathrm{NH}$ and $\mathrm{SH}$. The high-latitude NH has an amplitude that falls off with height, while in the SH the amplitude is more vertically coherent and less variable overall. One difference between these two regions is the amount of land. Land comprises most of each latitude band poleward of $45^{\circ} \mathrm{N}$, while elsewhere it is mostly ocean (ignoring Antarctica) as in Fig. 3. The reanalysis and models agree well on these features as shown in Fig. 4f.

\section{Projected changes}

Beginning around the second half of the twentieth century and continuing through the twenty-first century, 
(a) ERA-40 Phase
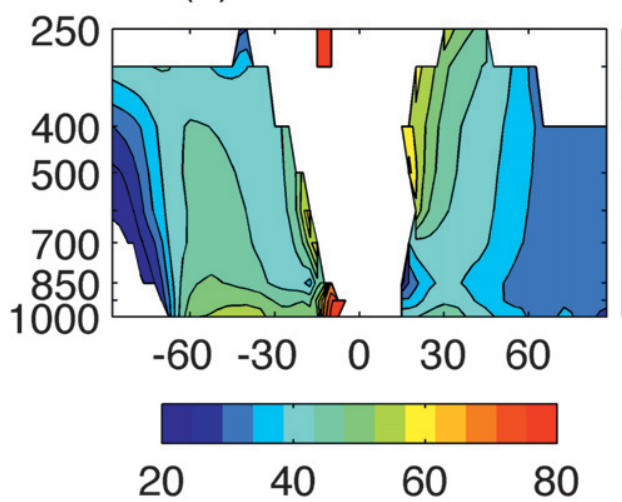

(d) ERA-40 Amplitude

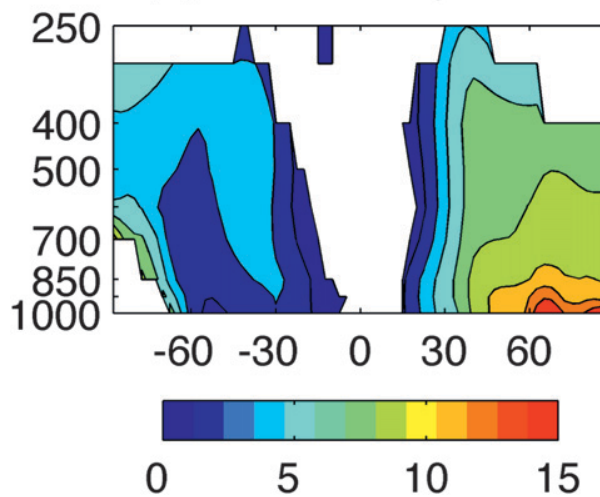

(b) CMIP3 Phase
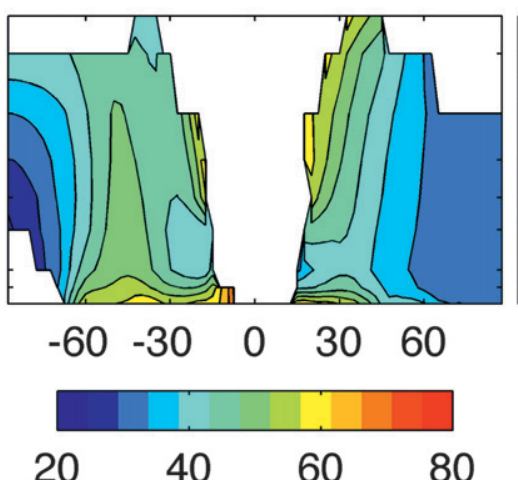

(e) CMIP3 Amplitude

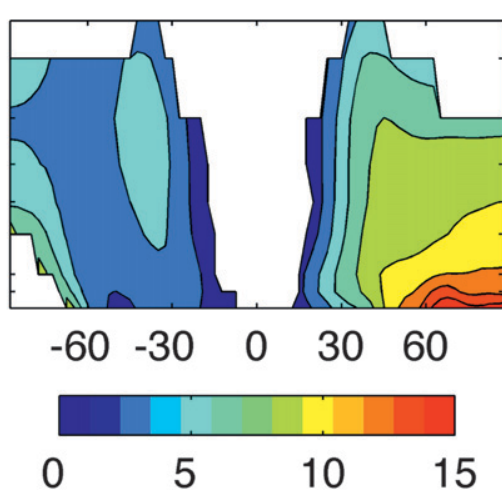

(c) CMIP3-ERA-40 Phase

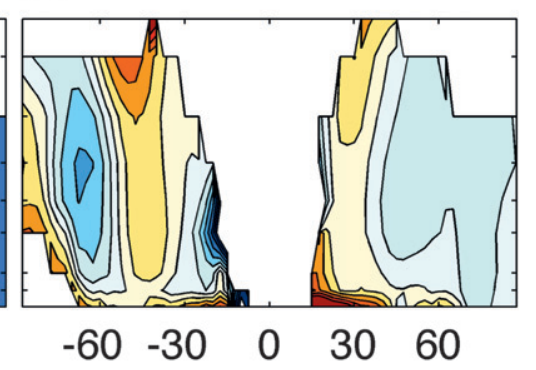

$-10$

0

10

(f) CMIP3-ERA-40 Amp.
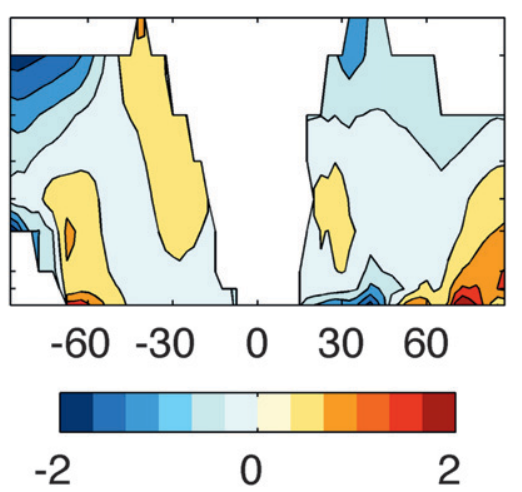

FIG. 4. Climatological mean seasonality, as in Fig. 2, but for zonally averaged tropospheric temperature aloft: (top) the phase for (a) ERA-40, (b) CMIP3, and (c) their difference and (bottom) the amplitude for (d) ERA-40, (e) CMIP3, and (f) their difference. In addition to ignoring locations where the annual cycle is weak, we do not plot the annual cycle in the stratosphere.

the models simulate a roughly linear increase in the global mean surface temperature phase lag from insolation and a linear decrease in the amplitude. These global changes are present for each of the 24 Special Report on Emissions Scenarios (SRES) A1B CMIP3 simulations in the time series of phase (Fig. 5a) and amplitude (Fig. 5b). The changes over land are smaller and less robust than those over ocean, consistent with the idea that sea ice loss is driving much of the change, as discussed in section $5 \mathrm{a}$. Over the ocean, the interannual variability is smaller than the change over the twenty-first century, for both phase and amplitude.

\section{a. Surface}

Where a change in effective heat capacity is the dominant mechanism altering the annual cycle of surface temperature, changes in phase and amplitude are constrained to be of the opposite sign. For example, if the effective heat capacity increases, the phase will shift to later in the year and the amplitude will decrease. On the other hand, in any region where there are changes in phase and amplitude that are not of opposite sign, changes in effective heat capacity are most likely not the primary driver.

The projected annual cycle changes in the twentyfirst century are consistent with an effective heat capacity increase in regions of large climatological sea ice cover. Figures $6 a$ and $6 \mathrm{~b}$ show latitude-longitude maps of the multimodel-mean projected temperature phase and amplitude changes between the last two decades of the twenty-first century and the last two decades of the twentieth century. The largest changes are over the high-latitude ocean with prominent sea ice, including the entire Arctic Ocean and Weddell and Ross Seas of Antarctica. Changes in these regions are robust: at least $75 \%$ of models agree with the multimodel mean on the sign of these changes (as indicated by the stippling).

Near the poles, the phase delay and amplitude decrease are much larger over ocean than land. For example, the delays in Greenland, northern Canada, and the Antarctic coast are all smaller than the delays over the ocean at the same latitude. The same holds true for amplitude, as we would expect from an effective heat 
(a) Temperature Phase

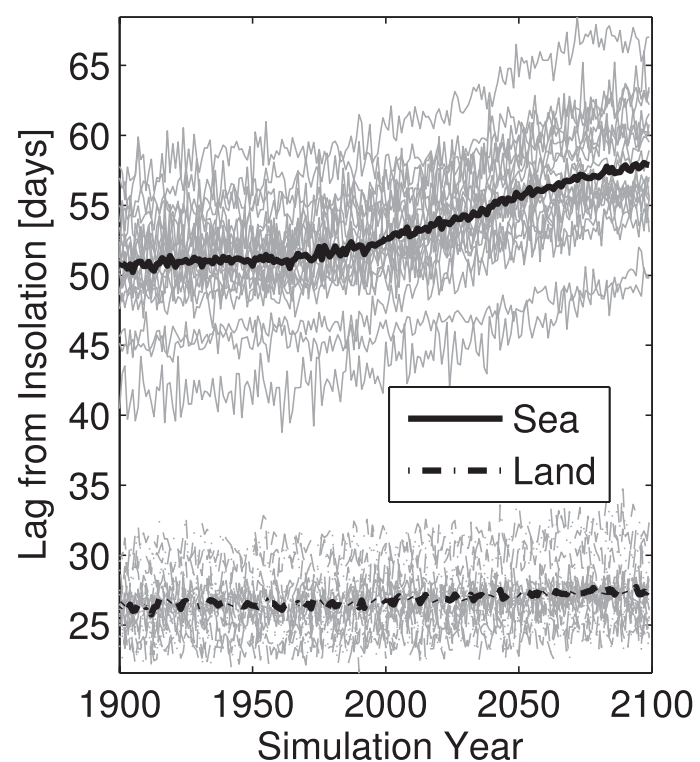

(b) Temperature Amplitude

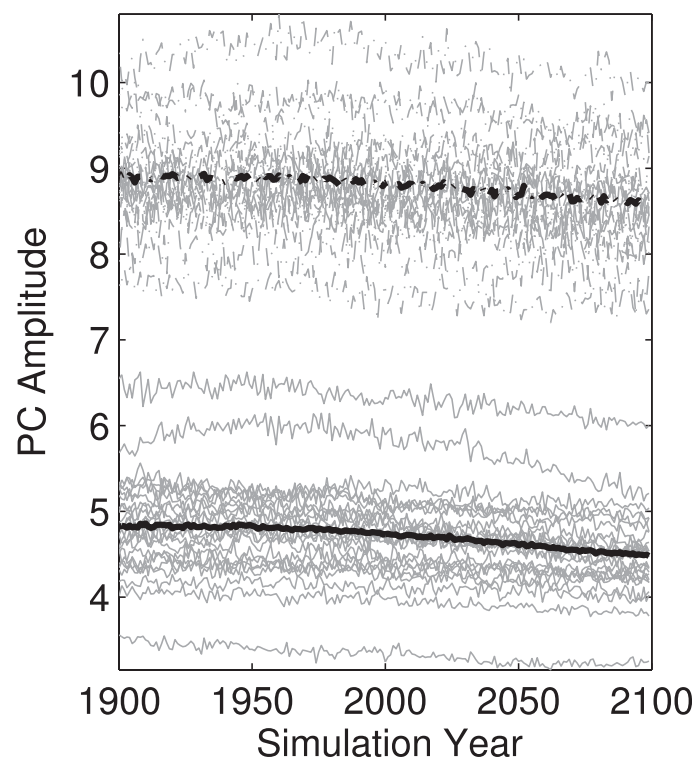

FIG. 5. Time series of the global surface air temperature (a) lag from insolation and (b) amplitude calculated with EOFs for all 24 models in the $20 \mathrm{C} 3 \mathrm{M}$ and A1B scenarios. The multimodel mean is in thick black and individual models are in gray. The solid lines represent the seasonality changes over ocean and the dashed-dotted lines represent the changes over land.

capacity increase over ocean. The largest changes over high-latitude land are near the coast.

The phase delays in the tropics and subtropics are much smaller than those at high latitudes, and there are actually several regions of phase advance. There is no discernible land-sea contrast in the low and midlatitudes, suggesting that the homogenous delay is not solely due to ocean heat capacity. Contrary to the phase change pattern, amplitude changes in the subtropics show a clear large-scale change in the opposite direction from that in the high latitudes: there is an amplitude increase of around $5 \%$ equatorward of $45^{\circ}$, most pronounced over ocean regions. This amplitude increase is not as large as the polar amplitude decrease, even after weighting by area. Yet this increase is prevalent among the models, especially in the NH. In the deep tropics, where the semiannual harmonic captures a large share of the total variance, the amplitude of the second harmonic also (a) Temperature Phase Change

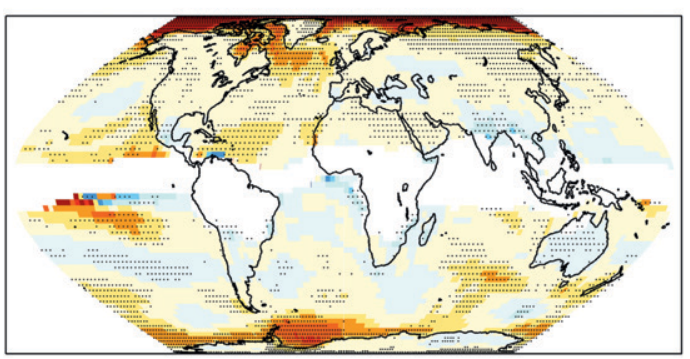

(b) Temperature Amp. Change
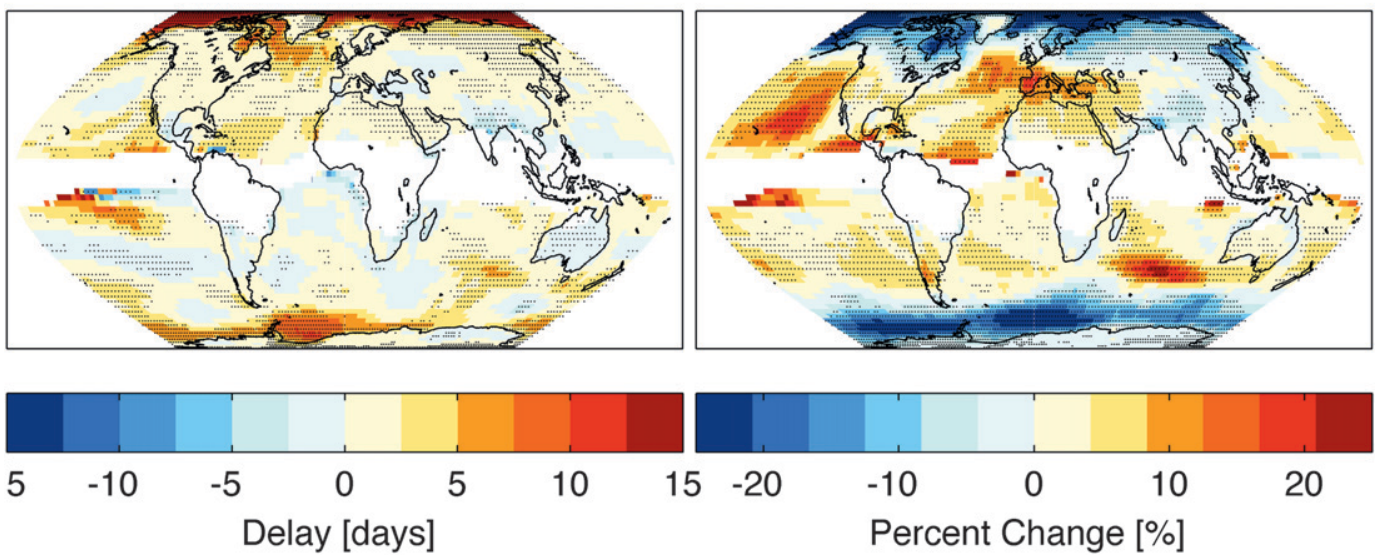

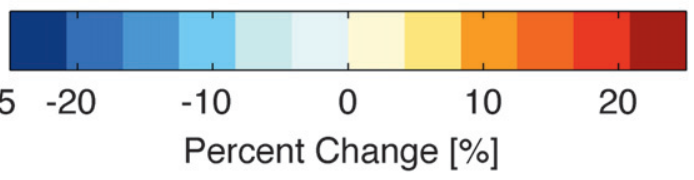

FIG. 6. The CMIP3 multimodel-mean annual surface temperature (a) phase and (b) amplitude change between 2080-99 and 1980-99. Stippling indicates that at least $75 \%$ of the models share the same sign as the mean change at that particular location. 
(a) Temperature Phase Change [days]

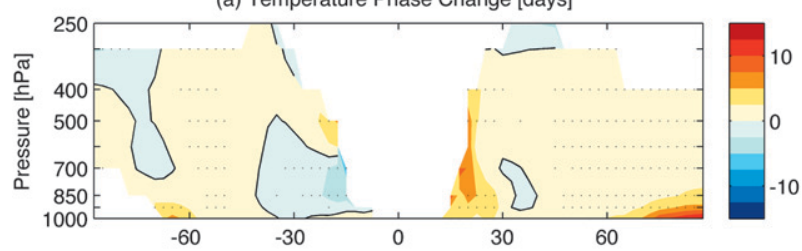

(b) Temperature Amplitude Change [\%]

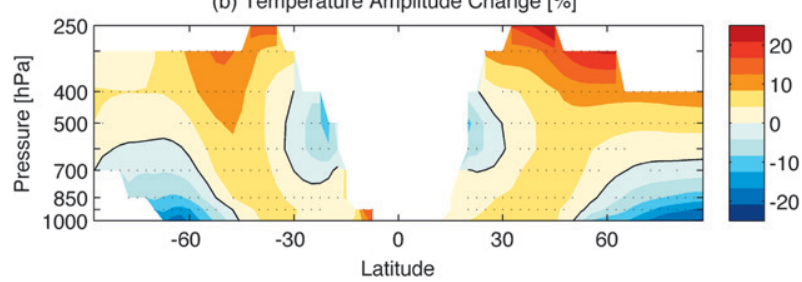

FIG. 7. As in Fig. 6, but for tropospheric seasonality changes for (a) phase and (b) amplitude.

increases by $15 \%-20 \%$ with the largest changes in the western Pacific Ocean (not shown). In between the low and high latitude responses (around $45^{\circ}-60^{\circ}$ in each hemisphere) is a transition zone where the amplitude change is small. In any individual model, the region of change is smaller, but averaging over all of the models enlarges the transition zone.

\section{b. Aloft}

The polar phase and amplitude changes are largest near the surface and weaken aloft, as shown in Figs. 7a and $7 \mathrm{~b}$. This is what we would expect for annual cycle changes controlled by surface characteristics, and it supports the idea that the surface temperature phase delay and amplitude reduction at the high latitudes are caused by an increased effective surface heat capacity, as first suggested by Manabe and Stouffer (1980). In fact, Kumar et al. (2010) found a similar seasonal, spatial warming structure aloft in a model simulation with prescribed sea ice loss. The polar changes are likely limited to the lower atmosphere because of the lack of deep vertical mixing due to the strong local atmospheric stability, but we note that, while the large surface phase delays are confined to the boundary layer and do not extend above $850 \mathrm{hPa}$, the amplitude reduction extends to $\sim 600 \mathrm{hPa}$.

Away from the polar surface, most of the troposphere shows a small phase delay of 1-2 days in the temperature cycle, of the same sign and similar in strength to the mean phase changes at the surface in the midlatitudes. Even though this delay is small, it is present in most models throughout the high-latitude $\mathrm{NH}$ troposphere. In the subtropical midtroposphere, there are amplitude decreases in both hemispheres that appear to be independent from changes at the surface. Aside from these regions, the rest of the troposphere has an amplitude increase, which is stronger still in the midlatitude stratosphere (both in relative and absolute magnitude). A. Donohoe and D. S. Battisti (2012, personal communication) argue that this amplitude increase is due to an increase in absorbed shortwave radiation by the atmosphere in summer mainly because of increased water vapor. There is an impressive amount of symmetry in the amplitude changes, considering that the climatological amplitude is not particularly symmetric. The changes are also robust in most locations except in regions where they reverse sign.

\section{Mechanisms}

To understand the high- and low-latitude seasonality changes in a more quantitative manner, we find it useful to analyze them in terms of a very simple model of the basic energy balance at the surface:

$$
C \frac{d T}{d t}=F[t, T(t)],
$$

where $C$ is the effective heat capacity, $T$ is temperature, and $F$ is net heat flux into the surface. Even though $C$ has a seasonal dependence due to changing mixed layer depths, sea ice, soil moisture, and other effects, we treat it as a constant for each period. This is both for the sake of simplicity and because the results of interest prove insensitive to the particulars of a seasonally varying heat capacity, once the annual mean value is specified. ${ }^{1}$

To isolate the factors that can affect the seasonal temperature cycle, we partition the net flux as $F[t, T(t)]=$ $Q(t)-\beta T$, where $Q(t)$ is the seasonal surface flux not linearly related to temperature (such as solar radiation) and $\beta$ is a constant. Physically, $-\beta T$ represents longwave flux, turbulent heat fluxes, and meridional heat transports to the extent that those damp the temperature response to $Q(t)$.

After Fourier transforming we find the following relation for the annual harmonic $\left(\omega=2 \pi \mathrm{yr}^{-1}\right)$ of $T$ and $Q$ :

$$
\begin{aligned}
i \omega C T & =Q-\beta T \\
T(\beta+i \omega C) & =Q,
\end{aligned}
$$

which yields the following phase and amplitude relations between $T$ and $Q$ :

$$
\begin{aligned}
\phi_{T}-\phi_{Q} & =\arctan \frac{\omega C}{\beta}, \\
|T| & =\frac{|Q|}{\sqrt{\beta^{2}+\omega^{2} C^{2}}} .
\end{aligned}
$$

\footnotetext{
${ }^{1}$ This was verified by numerically solving the temperature equation with a sinusoidally varying $C(t)$ with different phases.
} 
(a) Surface Flux Phase Change
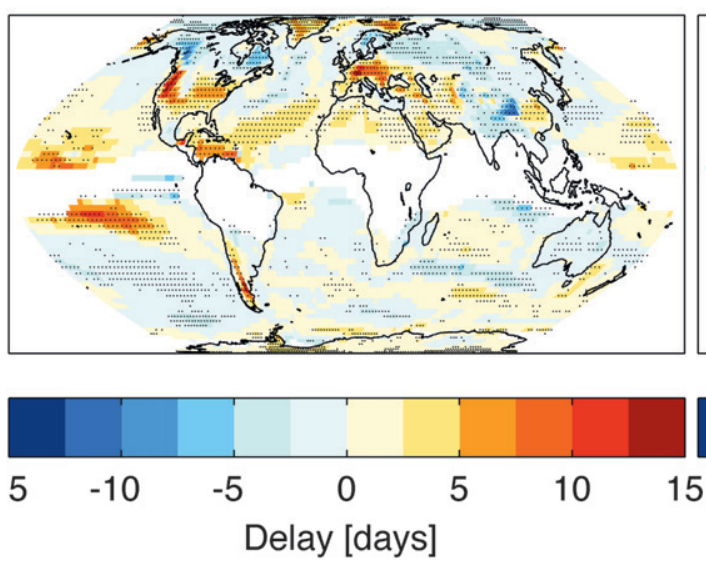

(b) Surface Flux Amp. Change
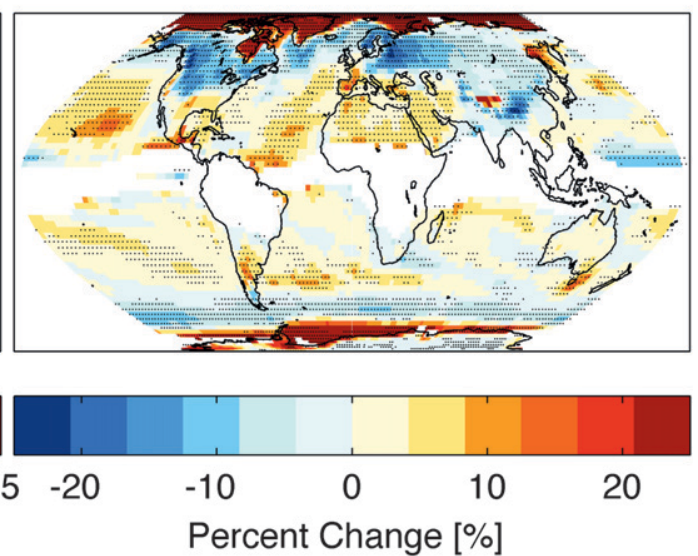

FIG. 8. As in Fig. 6, but for total surface flux change. Only locations where the annual cycle of surface flux represents at least $70 \%$ of the total variance are plotted.

The temperature phase lag is set by the ratio of $C$ to $\beta$. In the limiting case of small heat capacity, for which $\omega C /$ $\beta \rightarrow 0$, the temperature is in phase with $Q$, while for very large heat capacity $\omega C / \beta \rightarrow \infty$ and the temperature is in quadrature with $Q$. The relative amplitude of $T$ to $Q$ is inversely related to both $C$ and $\beta$. To understand the effects of changes in $C$ and $\beta$ we linearize Eq. (3) to find

$\Delta \phi_{T}-\Delta \phi_{Q}=\frac{\omega C / \beta}{1+(\omega C / \beta)^{2}}\left(\frac{\Delta C}{C}-\frac{\Delta \beta}{\beta}\right)$,

$\frac{\Delta|T|}{|T|}-\frac{\Delta|Q|}{|Q|}=\frac{-1}{1+(\omega C / \beta)^{2}}\left[\left(\frac{\omega C}{\beta}\right)^{2} \frac{\Delta C}{C}+\frac{\Delta \beta}{\beta}\right]$.

Assuming small variations in $\beta$ and $\phi_{Q}$, an increase in heat capacity will cause a phase delay. Likewise, for small variations in $\beta$ and $|Q|$, an increase in $C$ will lead to a decreased amplitude. Thus, we see that heat capacity changes have opposite effects on phase and amplitude and that, if phase and amplitude do not change in opposite ways, this implies that effective heat capacity changes are not the dominant effect. We can quantify this: since $(\omega C / \beta)^{2}$ is around 0.5 in the models, variations in $C$ are dominant when $\Delta C / C \gg 2 \Delta \beta / \beta$.

We have seen in the previous section that at high latitudes, changes are qualitatively consistent with an increase in effective heat capacity in regions where sea ice decreases in extent, thins, or becomes less persistent throughout the year. Below, we make this connection in more quantitative detail.

In the tropics and subtropics, both phase and amplitude increase and must therefore be forced at least in part by something other than changes in effective heat capacity. Below we provide evidence that this may be a consequence of a fractional increase in $\beta$ that is nearly an order of magnitude larger than the local fractional reduction in effective heat capacity.

\section{a. High latitudes}

Before demonstrating that the high-latitude phase delay and amplitude decrease of surface temperature are due to sea ice loss, we demonstrate that they are not directly due to changes in the surface flux cycle. For the flux to be responsible for the high-latitude seasonality changes to temperature, seasonal surface flux would need to lag peak insolation and weaken. In fact, the reverse happens, as shown in Figs. $8 \mathrm{a}$ and $8 \mathrm{~b}$.

There is little change in the phase of surface flux at high latitudes. In fact, the phase actually advances over some high-latitude ocean regions, indicating that the high-latitude temperature phase delay is not driven by changes in the surface flux phase. The surface flux amplitude, on the other hand, does show robust changes in the high latitudes. These changes, however, are of the opposite sign to the temperature amplitude changes. Over high-latitude ocean in both hemispheres, the surface flux amplitude increases by around $50 \%$ in both hemispheres. The increase is confined to the ocean and is in the same region as the reduction in the amplitude of surface temperature. Since the phase and amplitude changes of surface flux are of opposite sign to the temperature changes, they cannot be responsible for the latter.

The surface flux amplitude changes at high latitudes in Fig. 8b are consistent with sea ice loss (Screen and Simmonds 2010). Climatologically, the ice margin is not only the region of greatest upward turbulent heat flux during the winter but also where the total surface flux amplitude is greatest. As the ice edge shifts poleward during the twenty-first century, the consequence to the surface flux is an increase in amplitude in the polar 
ocean and a decrease in amplitude in the subpolar ocean (Deser et al. 2010). In the polar ocean the albedo is also reduced, which increases the downward shortwave radiation at the surface during the summer, contributing to an increased surface flux amplitude at high latitudes.

In terms of our energy balance model, Figs. $8 \mathrm{a}$ and $8 \mathrm{~b}$ show the seasonality changes to $F=Q-\beta T$. The seasonality changes in $Q(t)$ are similar to those in $F(t)$ at high latitudes. Whether we take $Q(t)$ to be the net shortwave flux at the surface or at the top of the atmosphere, we find the same small phase advance and large amplitude increase in the high latitudes (not shown). Hence, we can rule out seasonal changes of $Q$ as responsible for driving the high-latitude seasonal temperature changes.

In the multimodel mean, the surface temperature has a phase delay and amplitude decrease at high latitudes, consistent with an effective heat capacity increase. We also look for this consistency on an individual model basis. For example, do models with large phase delays also tend to have large amplitude decreases? We address this question in Fig. 9. On the $y$ axis we plot $\Delta \phi=\Delta \phi_{T}-$ $\Delta \phi_{Q}$, the change in the phase of the surface temperature relative to the change in phase of seasonal surface flux, and on the $x$ axis we plot $\Delta A=\Delta(|T| /|Q|)$, the change in the ratio of amplitudes of surface temperature to seasonal surface flux. The phase and amplitude changes are averaged over the $\mathrm{NH}$ and $\mathrm{SH}$ oceanic polar caps for each model. We find correlations between the phase and amplitude changes for both polar caps: $r=-0.67$ in the $\mathrm{NH}$ and $r=-0.79$ in the SH. Results are similar if we plot $\Delta \phi_{T}$ against $\Delta|T|$, though the correlations strengthen to $r=-0.79$ in the $\mathrm{NH}$ and weaken to $r=$ 0.17 in the SH. There is only one model where $\Delta \phi$ and $\Delta A$ have the same sign, and both phase and amplitude changes in that model are small.

Based on our energy balance model, we can calculate a theoretical relationship between phase and amplitude changes, assuming heat capacity changes while $\beta$ stays fixed. Because Fig. 9 shows a roughly linear relationship between the phase and amplitude changes, we use the linearized relationships of Eq. (4) and obtain a theoretical slope of $-\beta \sqrt{1+(\beta / \omega C)^{2}}$, though we might not expect a linear relationship because the changes are not small percentagewise (more than $25 \%$ for amplitude and $50 \%$ for phase since a 15 -day delay is half of the 30-day lag from insolation near the poles). We plot the multimodel mean of the theoretical slope with a dotted line in Fig. 9. The theoretical slope $(-0.08)$ is much flatter than the slope of the best-fit line $(-0.40)$ in the $\mathrm{NH}$, while for the $\mathrm{SH}$ the slopes are more similar $(-0.16$ theoretically and -0.26 for the best-fit line). We do not completely understand why the theoretical and best-fit slopes differ so much in the NH. An obvious possibility is that our very

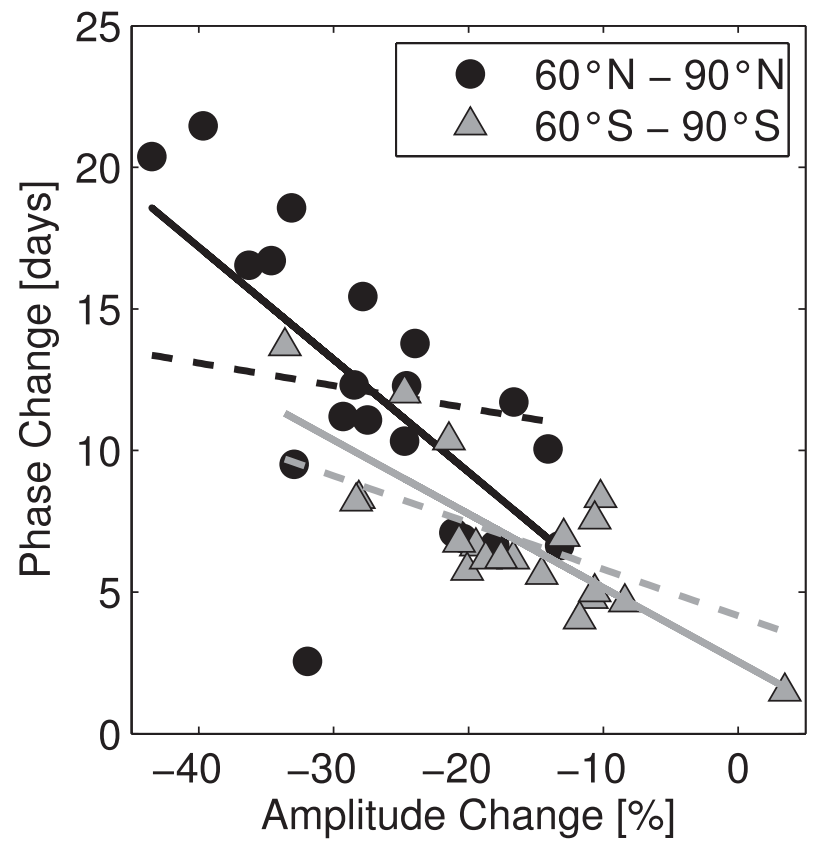

FIG. 9. Scatterplot of phase $\left(\phi_{T}-\phi_{Q}\right)$ and amplitude $|T| /|Q|$ changes for the $\mathrm{NH}$ (black circles) and $\mathrm{SH}$ (gray triangles) oceanic polar caps of the CMIP3 models between the periods 1980-99 and 2080-99. Each pair of black and gray markers represents a single model. The solid lines are the least squares best-fit line, and the dashed lines describe the theoretically predicted slopes as described in section 5 a.

simple, zero-dimensional, two-parameter model is inadequate to capture the GCM behavior at this quantitative level; another is that the multimodel mean is not the most appropriate estimate of $\beta$ for use to compute the theoretical slope. Nonetheless, a change in $\beta$ alone would produce a positive correlation, and the fact that the changes in phase and amplitude are negatively correlated qualitatively supports the hypothesis that heat capacity changes control the seasonality changes.

To further quantify the extent to which seasonality changes are due to $C$ or $\beta$, we calculate the changes to effective heat capacity in the context of our energy balance model. Solving Eq. (3) for $C$ and $\beta$ in terms of phase and amplitude gives the following:

$$
C=\frac{\sin \phi}{\omega A}, \quad \beta=\frac{\cos \phi}{A} .
$$

Since we calculate $A$ and $\phi$ directly via Fourier transform, Eq. (5) gives expressions for the $C$ and $\beta$ changes for the CMIP3 models in the context of this simple temperature model.

In our calculations we take $Q$ to be the net shortwave flux at the surface, but the results are nearly the same if we take it to be the net shortwave flux at the top of the 
(a) Polar: $60^{\circ}-90^{\circ}$

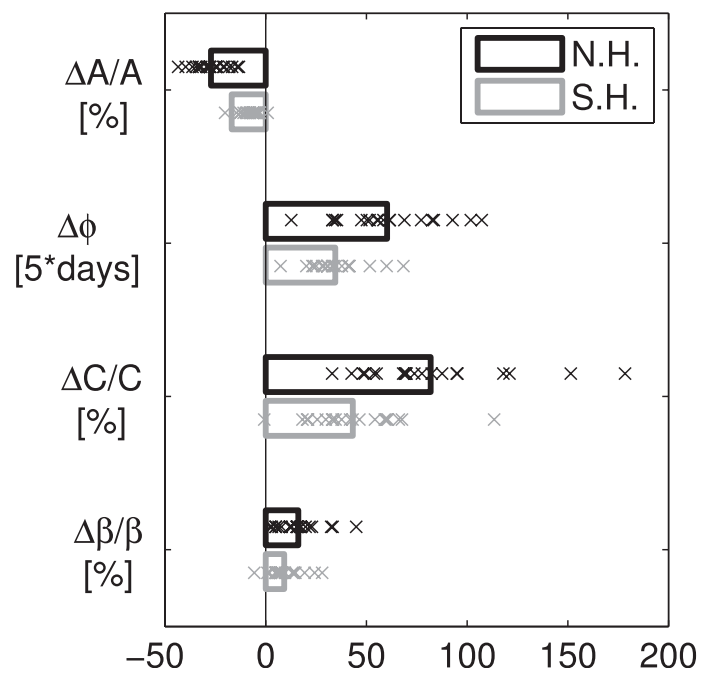

(b) Subtropical: $15^{\circ}-30^{\circ}$

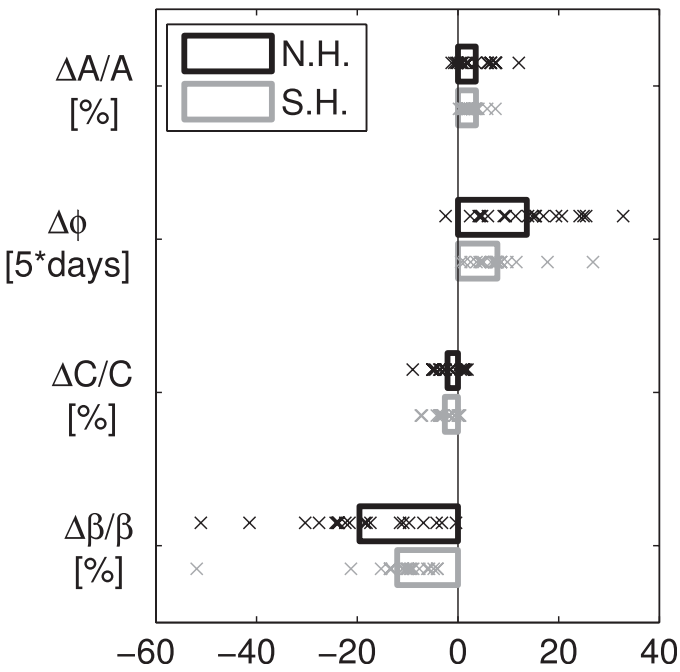

FIG. 10. Changes in amplitude, phase, effective heat capacity, and $\beta$ for the NH (black) and SH (gray) in (a) high latitudes and (b) low latitudes over ocean for each CMIP3 model. The multimodel mean is represented by a bar and individual models by " $\times$." The amplitude and phase are found from a Fourier transform, and the effective heat capacity and $\beta$ are found from Eq. (5). The changes in phase have been multiplied by 5 to use the same axis for all quantities. Note that the scale of (a) is 2.5 times that of (b).

atmosphere. For both surface temperature and net surface shortwave flux we calculate the average phase and amplitude over the ocean poleward of $60^{\circ}$ for each hemisphere for the last two decades of the twentieth and twenty-first centuries. From these values we find $C$ and $\beta$ and plot the changes in Fig. 10a.

Changes to $C$ show a robust increase across the multimodel ensemble in both hemispheres: nearly every single model predicts an increase in effective heat capacity. The multimodel mean increases are $82 \%$ and $43 \%$ for the $\mathrm{NH}$ and $\mathrm{SH}$, respectively. Changes to $\beta$ are smaller but also positive for nearly all of the models. The multimodelmean increase is $16 \%$ for the $\mathrm{NH}$ and $9 \%$ for the $\mathrm{SH}$. We interpret the $\beta$ changes mathematically as an increased damping in the system, and physically as the turbulent and longwave fluxes and heat transports-in some combination-becoming more effective at returning the surface temperature to equilibrium. Which of the processes involved is most responsible for this change and how the change is ultimately forced by greenhouse gas increases is not yet clear and will require further study.

Despite the robust increase in $\beta$, the proportionally larger increase in $C$ has the greater influence on the changes in seasonality of temperature at high latitudes. From Eq. (4), phase delays are proportional to $\Delta C / C-$ $\Delta \beta / \beta$, indicating that, if $\beta$ did not change, the phase delay would be even larger. Amplitude changes are proportional to $-\Delta \beta / \beta-(\omega C / \beta)^{2} \Delta C / C$, where $(\omega C / \beta)^{2}$ is a proportionality factor averaging 0.4 for the $\mathrm{NH}$ and
0.7 for the SH in the twentieth century. Since both $\beta$ and $C$ increase in the twenty-first century, both are responsible for a decreased amplitude. However, when we calculate the multimodel mean of $(\omega C / \beta)^{2}(\Delta C / C)$ $(\Delta \beta / \beta)^{-1}$, we find that the contribution from the heat capacity change term is two to three times larger than that from the $\beta$ term.

Sea ice loss was postulated to be the reason for highlatitude changes in seasonality by earlier authors (Manabe and Stouffer 1980; Manabe et al. 1992; Mann and Park 1996). The explanation goes as follows: sea ice acts as a partition between the atmosphere and ocean by shutting off radiative transfer and turbulent heat fluxes between them. The only coupling is by conduction through the sea ice. As sea ice melts, the insulating effect wanes and the ocean and atmosphere can more freely exchange heat, raising the effective heat capacity of the surface. Any external addition of heat, such as from solar radiation, will more easily be shared between the atmosphere and ocean if there is less sea ice. Sea ice loss is robust in the models: sea ice area diminishes in every model at a roughly linear rate during the twenty-first century (Fig. 11). The NH suffers a larger ice loss than the $\mathrm{SH}$, which may partly account for why the amplitude and phase changes are larger in the $\mathrm{NH}$.

If all of the effective heat capacity increase were due to sea ice loss, then $\Delta C / C$ would be roughly proportional to the fractional change in the open ocean area. We calculate the latter quantity for each model 
(a) Northern Hemisphere

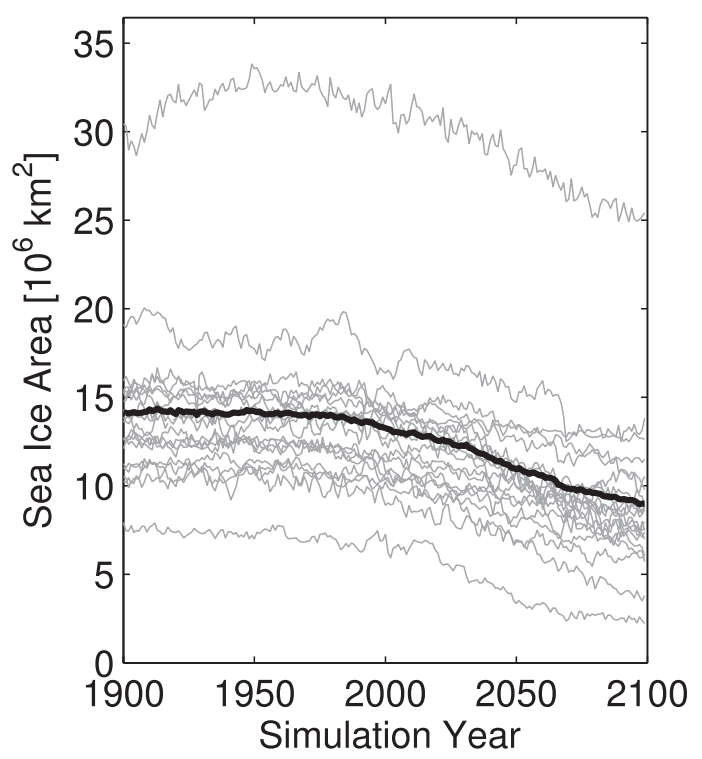

(b) Southern Hemisphere

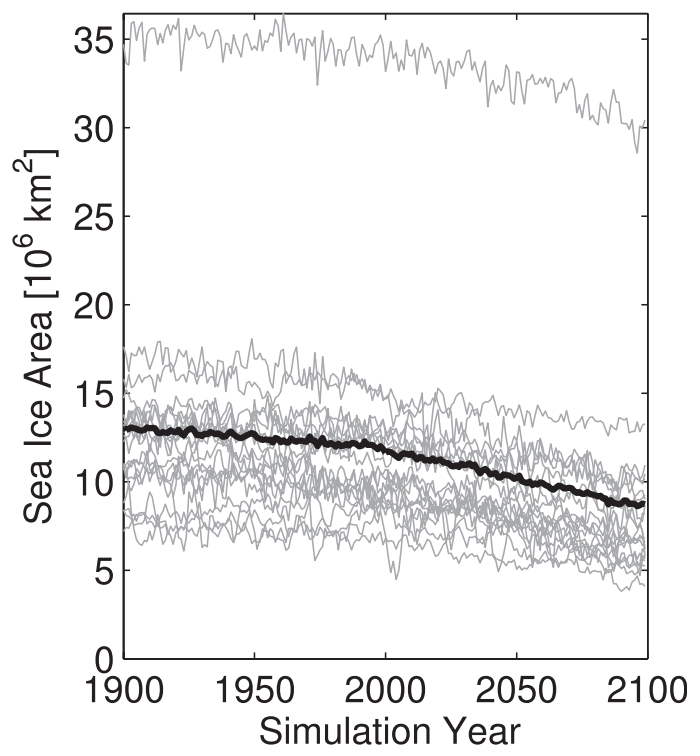

FIG. 11. Time series of annually averaged sea ice area in the (a) $\mathrm{NH}$ and (b) SH polar caps $\left(60^{\circ}-90^{\circ}\right)$ for the CMIP3 models. The thick black line is the multimodel mean.

and in Fig. 12 plot it against the fractional change in effective heat capacity for each model as calculated from Eq. (5).

The two calculations of effective heat capacity correlate well with each other, indicating that sea ice loss is probably the dominant mechanism for the effective heat capacity change. While the correlations are strong, the models do exhibit a bias: the two effective heat capacity calculations are not randomly distributed about the one-to-one line, but instead the effective heat capacity change is larger when calculated from Eq. (5). This could be due to the limitations of relating effective heat capacity increase to ice area alone. For example, simply measuring the open ocean increase does not take into account sea ice thinning, which would increase the effective heat capacity relative to a thick sea ice layer. Regardless, sea ice area loss appears to account for most of the effective heat capacity increase that is driving the delayed and weakened annual temperature cycle in the high latitudes.

Another way to quantify the relationship between temperature annual cycle changes and sea ice changes is to correlate the two across models in the ensemble. We focus on high-latitude (poleward of $60^{\circ}$ ) annual cycle changes to air temperature over ocean so as to determine if models with large sea ice loss tend to have large phase delays and weak annual cycles. We find that correlations of temperature phase delay with annual sea ice area change are significant for the $\mathrm{NH}(r=-0.67)$ but not for the SH $(r=-0.35)$ at the $95 \%$ level (Fig. 13a).
Correlations between amplitude change and sea ice area change are $r=0.51$ for the $\mathrm{NH}$ and $r=0.46$ for the $\mathrm{SH}$ and are significant for both hemispheres (Fig. 13b). These correlations do not significantly change if we weight the area loss with a factor to account for the reduction of ice thickness.

\section{b. Low latitudes}

While sea ice loss seems to explain the high-latitude phase and amplitude changes of the annual temperature cycle, it does not directly explain the changes at low latitudes. Equatorward of roughly $45^{\circ}$ the models simulate a slight phase delay and an increased amplitude. One possible explanation for this behavior is that the high-latitude seasonality changes are transported equatorward, for example, by midlatitude eddies. There are two reasons why this is unlikely. For one, the amplitude increases at low latitudes, while it decreases near the poles. The other reason is that models that have large delays in the high latitudes do not tend to have large delays in the subtropics. The only region-to-region phase correlations that appear significant are between the extratropics and subtropics in the Northern Hemisphere $(r=0.59)$. But the amplitude correlations are small between these two regions $(r=0.17)$, suggesting that the delay in the low latitudes is not simply communicated from higher latitudes.

An alternate explanation is that the temperature seasonality changes are a result of surface flux seasonality changes. While the phase and amplitude of surface flux 


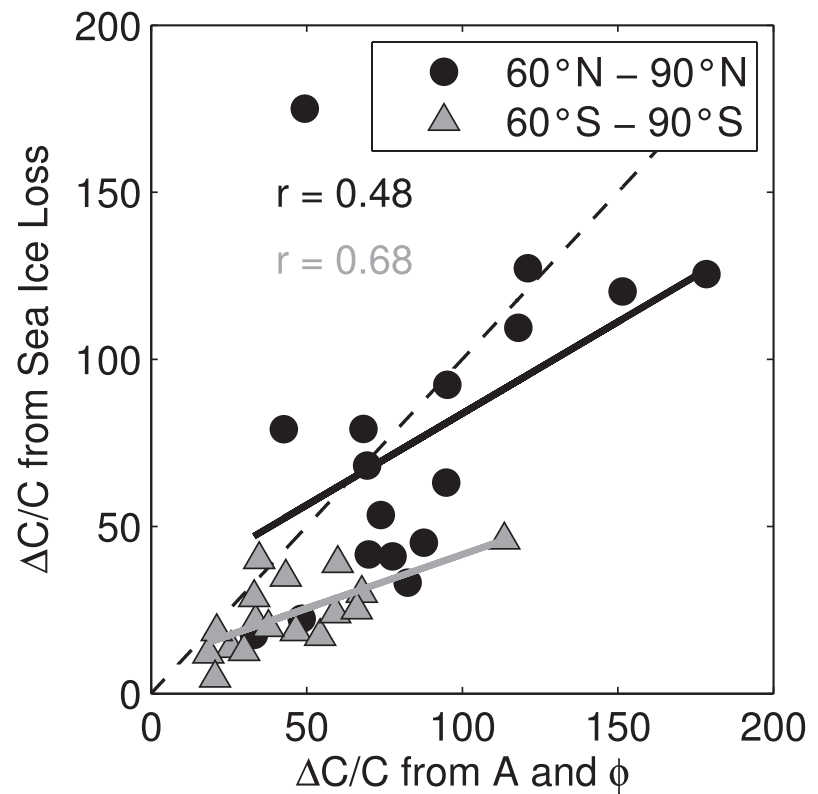

FIG. 12. Scatterplot of fractional effective heat capacity changes in the CMIP3 models calculated from the increase in open ocean fraction on the $y$ axis and from the amplitude and phase on the $x$ axis for the NH (black circles) and SH (gray triangles) polar caps. Each marker represents an individual model, and the dashed line is the one-to-one correspondence between the axes. Correlations are $r=0.48$ for the NH and $r=0.68$ for the SH. The slopes of the lines are 0.55 for the $\mathrm{NH}$ and 0.32 for the $\mathrm{SH}$.

changes are opposite in sign to those of the temperature changes at high latitudes, this is not the case at low latitudes, as shown in Fig. 8. The phase of both surface temperature and surface flux show a small delay-less than 5 days-from $45^{\circ} \mathrm{S}$ to $45^{\circ} \mathrm{N}$. The amplitude changes of temperature and surface flux are even more similar. From $45^{\circ} \mathrm{S}$ to $45^{\circ} \mathrm{N}$ both temperature and surface flux amplitude show broad increases of around $5 \%$.

There is also a strong spatial correlation between the temperature and flux changes. Phase delays occur in the same places, such as the eastern Pacific and the $\mathrm{NH}$ subtropical Atlantic. Both the temperature and flux also have especially large amplitude increases in the eastern Pacific and eastern Atlantic. We create a measure of spatial correlation in Fig. 14 by plotting the multimodel-mean seasonality changes for temperature and flux against one another for all subtropical ocean grid boxes between $15^{\circ}$ and $30^{\circ}$ in both hemispheres, excluding locations where the annual cycle is small. There are strong correlations of $r=0.67$ for the phases and $r=0.78$ for the amplitudes, indicating that the surface flux changes are spatially correlated with the surface temperature changes.

We can understand these changes in the context of our energy balance model. While the seasonal cycle of total
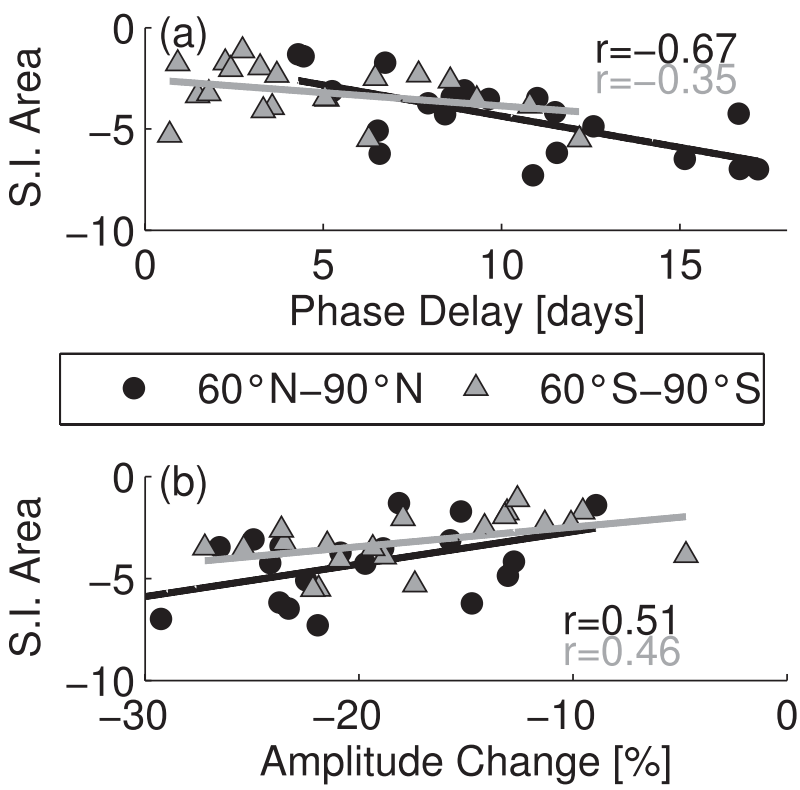

FIG. 13. Correlations between sea ice area loss and (a) temperature phase delay and (b) temperature amplitude change for the polar NH (black circles) and polar SH (gray triangles) in the CMIP3 models between 2080-99 and 1980-99.

flux $F$ delays and strengthens in the subtropics in Fig. 8, the same cannot be said of the temperature-independent component of the flux $Q$. There is almost no change in the phase or amplitude of the net shortwave radiation at the surface (not shown). Since $F=Q-\beta T$, this suggests that changes in $\beta$ are responsible for the changes in total surface flux.

To find the explicit changes to $C$ and $\beta$ we use the same procedure as for the high latitudes by calculating $C$ and $\beta$ from $A$ and $\phi$ with Eq. (5) and plot these results in Fig. 10b. The phase delay is around 2.8 days in the $\mathrm{NH}$ and 1.6 days in the $\mathrm{SH}$, while the amplitude increase is around $3 \%-4 \%$ for both Northern and Southern Hemispheres. While small, these changes are robust in the models: the vast majority have the same sign as the mean change. Unlike in the high latitudes, we find a small decrease in the subtropical heat capacity around $2 \%-3 \%$ in both hemispheres. This might be a consequence of a reduction in tropical ocean mixed layer depth (Philip and Oldenborgh 2006). The larger changes are in $\beta$, which decreases by $20 \%$ in the $\mathrm{NH}$ and $12 \%$ in the SH. Since $\Delta \phi \propto \Delta C / C-\Delta \beta / \beta$, we can attribute the subtropical phase delay primarily to the $\beta$ decrease. Likewise, since $\Delta A / A \propto-\Delta \beta / \beta-(\omega C / \beta)^{2} \Delta C / C$ and $\omega C / \beta<1$, the amplitude increase is also primarily due to the $\beta$ decrease.

The reduction in $\beta$ indicates that twenty-first-century temperature in the subtropics becomes more weakly damped. Physically, a weakened $\beta$ means that the 
(a) Phase Delay [days]

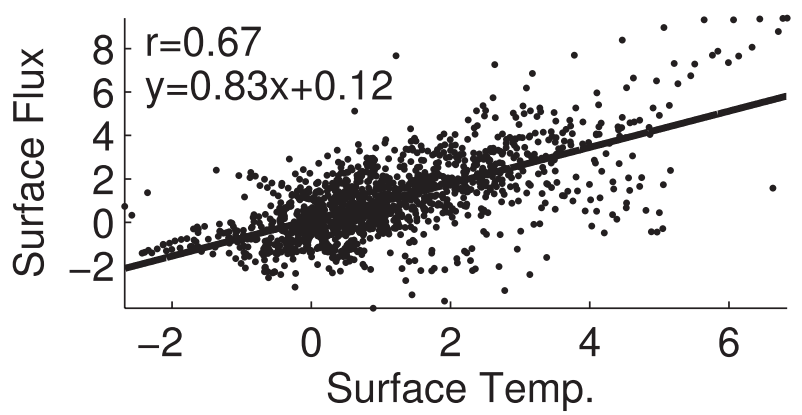

(b) Amplitude Change [\%]

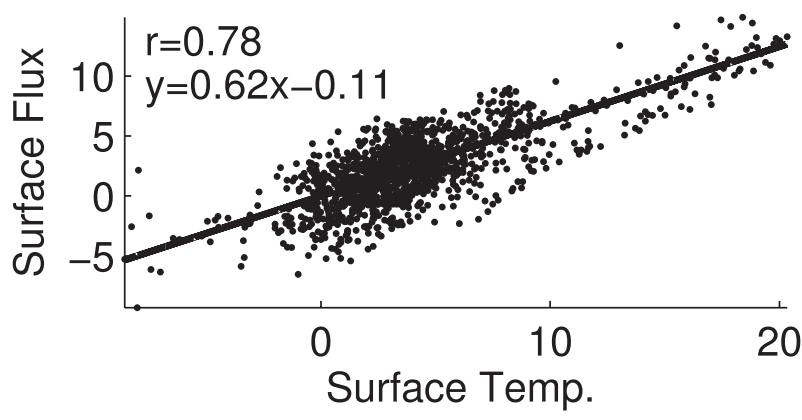

FIG. 14. Scatterplot of twenty-first-century seasonality changes of surface flux vs surface temperature for (a) phase and (b) amplitude for subtropical ocean grid boxes. There is an area-weighted correlation between these two variables for both phase and amplitude indicating that, e.g., subtropical locations that have large surface flux delays tend to have large surface temperature delays. The data is restricted to $15^{\circ}-30^{\circ}$ in both hemispheres and does not include locations where the annual harmonic of each variable does not dominate its total variance. The solid line represents the areaweighted least squares regression.

combination of turbulent and latent fluxes and heat transports become less effective at returning the surface temperature to equilibrium. A reduced $\beta$ does not necessarily imply a weakened surface flux amplitude because the total surface flux $F=Q-\beta T$ also depends on the phase relationship between $T$ and $Q$. For the low latitudes the surface flux amplitude increases despite the reduction in $\beta$. Sobel and Camargo (2011) presented evidence that the surface flux amplitude increases are driven by changes in the seasonal cycle of surface winds, with subtropical winds increasing in the winter hemisphere and decreasing in the summer hemisphere. At a fixed subtropical location, these changes in wind speed change sign over the year and thus will not be described well by a simple change in an otherwise constant coefficient $\beta$. Further, because surface air humidity (the other state variable that enters the bulk formula for the surface latent heat flux, besides wind speed and SST) can adjust so quickly to other factors, it may be appropriate to view these wind speed changes as an external forcing on the surface fluxes, rather than a change in a damping coefficient in an SST equation. These considerations suggest that the simple model we have proposed to interpret the high-latitude seasonality changes projected by the models may be inadequate to capture the lowlatitude changes. Further work is required to determine the exact roles of the surface wind and other factors in the surface energy budget. It is clear, however, that there is a link between the net surface heat flux and the seasonal temperature cycle at low latitudes, unlike at high latitudes where the effective heat capacity governs the changes in seasonality.

\section{Conclusions}

In this study we analyzed the changes to the seasonality of surface temperature in response to an increase in greenhouse gases during the twenty-first century as represented by the CMIP3 models. We found large, robust, global changes to the annual cycle of surface temperature: a phase delay and an amplitude reduction. By analyzing these changes geographically, we found that the phase delay and amplitude decrease are strongest at high latitudes and drive the global response. These polar changes are consistent with an effective heat capacity increase of the surface layer due to sea ice loss. At low latitudes there is a small phase delay and an amplitude increase, which we linked to changes in the seasonality of the surface heat flux.

CMIP3 climate models accurately represent the typical phase and amplitude of the annual cycle aloft and at the surface as represented by the ERA-40. While the two are not completely independent, since the reanalysis incorporates a climate model, the agreement is encouraging. Geographic variations in models and the reanalysis are spatially consistent and can be traced to different surface effective heat capacities: temperature over ocean responds slowly and weakly, while temperature over land and sea ice responds rapidly and strongly.

At high latitudes the temperature cycle delays and weakens in response to greenhouse gases in the CMIP3 models. We provided evidence that sea ice loss is driving these changes. By fitting CMIP3 data to a parameterized surface energy balance model, we found that an increase in effective heat capacity primarily accounts for the phase delay and amplitude decrease at high latitudes. We also demonstrated that the increase in effective heat capacity for each model was consistent with the increase in openocean fraction, indicating that sea ice loss is driving the effective heat capacity and seasonality changes at high latitudes. We provided further evidence of this mechanism by showing strong correlations between sea ice loss 
and phase and amplitude changes among the models at the high latitudes in each hemisphere.

The projected delayed and weakened temperature cycle in the high-latitudinal $\mathrm{NH}$ is a manifestation of Arctic amplification, the accelerated annual mean warming in the Arctic Ocean relative to the rest of the globe predicted by all CMIP3 A1B twenty-first-century climate simulations. Arctic amplification has a seasonal component to it as well, with models predicting little warming in summer and substantial warming during the late fall and early winter (Serreze and Francis 2006). This warming structure is consistent with the changes in the annual harmonic of phase and amplitude. While the models predict the surface annual cycle changes to grow over the course of the twenty-first century, recent studies have already found early signs of changes in the Arctic Ocean. Among four different datasets, Serreze et al. (2009) and Screen and Simmonds (2010) found evidence of a delayed and weakened temperature cycle in the Arctic Ocean, consistent with rapid sea ice loss over this period and providing support for future changes expected by the CMIP3 models.

We suggest that the high-latitude seasonal temperature changes are credible. Not only are they prevalent among the models but also linked to a clearly identifiable physical process in the models: sea ice loss. While there has been some disagreement between models and observations of temperature phase changes over the midlatitude $\mathrm{NH}$ land during the twentieth century (Stine et al. 2009), substantial sea ice loss is already occurring in the Arctic. Furthermore, trends of an Arctic temperature phase delay and amplitude decrease have been observed during the last 30 years.

Changes in the temperature cycle at low latitudes are different in nature than those at high latitudes. While still robust, they have a small phase delay and a small amplitude increase, inconsistent with an increase in effective heat capacity. However, the changes in both phase and amplitude are consistent with a delayed and strengthened surface flux cycle that we traced to a decrease in damping of surface temperature by turbulent and longwave heat fluxes in our energy balance model. We also found a strong spatial correlation between seasonality changes in surface flux and surface temperature in the subtropics. Sobel and Camargo (2011) describe a link between changes in the amplitude of the seasonal cycle in SST and those in surface wind speed and describe the latter as a consequence of the expansion of the Hadley cell. While we do not understand the mechanism responsible for the phase changes in the net surface flux, it is worth further effort to do so. Phase changes in tropical precipitation (Biasutti and Sobel 2009) may also be linked to the phase changes in tropical SST and will be the subject of future work.

Acknowledgments. We thank Ian Eisenman for discussions, Naomi Naik for help with the CMIP3 dataset, and three anonymous reviewers for their comments. This research was supported by NSF Grant AGS0946849 and NASA Earth and Space Science Fellowship NNX11AL88H. We acknowledge the modeling groups, the Program for Climate Model Diagnosis and Intercomparison (PCMDI) and the WCRP Working Group on Coupled Modeling (WGCM) for their roles in making available the WCRP CMIP3 multimodel dataset. Support of this dataset is provided by the Office of Science, U.S. Department of Energy. We thank ECMWF for providing the ERA-40 reanalysis dataset.

\section{REFERENCES}

Biasutti, M., and A. H. Sobel, 2009: Delayed Sahel rainfall and global seasonal cycle in a warmer climate. Geophys. Res. Lett., 36, L23707, doi:10.1029/2009GL041303.

Carson, J. E., and H. Moses, 1963: The annual and diurnal heatexchange cycles in upper layers of soil. J. Appl. Meteor., 2, 397-406.

Chiang, J. C. H., Y. Kushnir, and A. Giannini, 2002: Deconstructing Atlantic intertropical convergence zone variability: Influence of the local cross-equatorial sea surface temperature gradient and remote forcing from the eastern equatorial Pacific. J. Geophys. Res., 107, 4004, doi:10.1029/ 2000JD000307.

Deser, C., R. Tomas, M. Alexander, and D. Lawrence, 2010: The seasonal atmospheric response to projected Arctic sea ice loss in the late twenty-first century. J. Climate, 23, 333-351.

Eicken, H., 2003: From the microscopic, to the macroscopic, to the regional scale: Growth, microstructure and properties of sea ice. Sea Ice: An Introduction to its Physics, Chemistry, Biology and Geology, D. N. Thomas and G. S. Dieckmann, Eds., Blackwell Science Ltd., 22-81.

Fitter, A. H., and R. S. R. Fitter, 2002: Rapid changes in flowering time in British plants. Science, 296, 1689-1691, doi:10.1126/ science.1071617.

Kumar, A., and Coauthors, 2010: Contribution of sea ice loss to Arctic amplification. Geophys. Res. Lett., 37, L21701, doi:10.1029/ 2010GL045022.

Kutzbach, J. E., 1967: Empirical eigenvectors of sea-level pressure, surface temperature and precipitation complexes over North America. J. Appl. Meteor., 6, 791-802.

Loon, H. V., 1967: The half-yearly oscillations in middle and high southern latitudes and the coreless winter. J. Atmos. Sci., 24, 472-486.

Manabe, S., and R. J. Stouffer, 1980: Sensitivity of a global climate model to an increase of $\mathrm{CO}_{2}$ concentration in the atmosphere. J. Geophys. Res., 85 (C10), 5529-5554.

— M. J. Spelman, and R. J. Stouffer, 1992: Transient responses of a coupled ocean-atmosphere model to gradual changes of atmospheric $\mathrm{CO}_{2}$. Part II: Seasonal response. J. Climate, 5, $105-126$.

Mann, M. E., and J. Park, 1996: Greenhouse warming and changes in the seasonal cycle of temperature: Model versus observations. Geophys. Res. Lett., 23, 1111-1114. 
Meehl, G., C. Covey, T. Delworth, M. Latif, B. McAvaney, J. Mitchell, R. Stouffer, and K. Taylor, 2007: The WCRP CMIP3 multimodel dataset. Bull. Amer. Meteor. Soc., 88, 1383-1394.

Philip, S., and G. V. Oldenborgh, 2006: Shifts in ENSO coupling processes under global warming. Geophys. Res. Lett., 33, L11704, doi:10.1029/2006GL026196.

Screen, J. A., and I. Simmonds, 2010: Increasing fall-winter energy loss from the Arctic Ocean and its role in Arctic temperature amplification. Geophys. Res. Lett., 37, L16707, doi:10.1029/ 2010 GL044136.

Serreze, M. C., and J. A. Francis, 2006: The Arctic amplification debate. Climatic Change, 76, 241-264, doi:10.1007/s10584-005-9017-y.

- A. P. Barrett, J. C. Stroeve, D. N. Kindig, and M. M. Holland, 2009: The emergence of surface-based Arctic amplification. Cryosphere, 3, 11-19, doi:10.5194/tc-3-11-2009.

Seth, A., S. A. Rauscher, M. Rojas, A. Giannini, and S. J. Camargo, 2011: Enhanced spring convective barrier for monsoons in a warmer world? Climatic Change, 104, 403-414, doi:10.1007/ s10584-010-9973-8.
Sobel, A., and S. Camargo, 2011: Projected future seasonal changes in tropical summer climate. J. Climate, 24, 473-487.

Stewart, I., D. Cayan, and M. Dettinger, 2005: Changes toward earlier streamflow timing across western North America. J. Climate, 18, 1136-1155.

Stine, A. R., and P. Huybers, 2012: Changes in the seasonal cycle of temperature and atmospheric circulation. J. Climate, in press.

,-- , and I. Y. Fung, 2009: Changes in the phase of the annual cycle of surface temperature. Nature, 457, 435-440, doi:10.1038/nature07675.

Stroeve, J., M. M. Holland, W. Meier, T. Scambos, and M. Serreze, 2007: Arctic sea ice decline: Faster than forecast. Geophys. Res. Lett., 34, L09501, doi:10.1029/2007GL029703.

Thomson, D., 1995: The seasons, global temperature, and precession. Science, 268, 59-68.

Trenberth, K., 1983: What are the seasons? Bull. Amer. Meteor. Soc., 64, 1276-1277.

Uppala, S., and Coauthors, 2005: The ERA-40 Re-Analysis. Quart. J. Roy. Meteor. Soc., 131, 2961-3012, doi:10.1256/qj.04.176. 